\title{
Original rat model of high kinetic unilateral pulmonary hypertension surgically induced by combined surgery
}

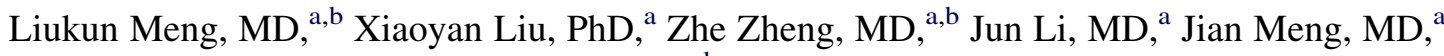 \\ Yingjie Wei, $\mathrm{PhD},{ }^{a}$ and Shengshou $\mathrm{Hu}, \mathrm{MD}^{\mathrm{a}, \mathrm{b}}$
}

\begin{abstract}
Objectives: The characteristic morphologic lesions observed in the lungs of patients with congenital cardiac anomalies have not been closely modeled in rat shunt-related models, except for the reversible grade 1 changes. The present study reported an original rat model of unilateral pulmonary hypertension surgically induced by combined surgery to reproduce more advanced pulmonary vascular lesions.
\end{abstract}

\begin{abstract}
Methods: The right pulmonary artery was ligated through a right posterolateral thoracotomy, and a cervical shunt was established 1 week later. The immediate and chronic effects on the pulmonary hemodynamics were evaluated through right heart catheterization immediately after and at 8 and 12 weeks postoperatively. The morphologic changes in pulmonary vasculature were analyzed after staining with hematoxylin-eosin and modified Weigert's method. The right ventricular hypertrophy index was calculated and artery blood gas analysis performed.
\end{abstract}

Results: A pulmonary hypertensive status was successfully induced immediately after cervical surgery and progressively aggravated into a borderline state with disease course advancing. Pulmonary vasculopathy demonstrated a transition from reversibility (muscularization, intimal proliferation of grade 1-2) at 8 weeks to irreversibility (intimal fibrosis, entirely luminal occlusion, grade 3 ) at 12 weeks postoperatively. Conspicuous right ventricular hypertrophy and decreasing partial arterial pressure of oxygen were also observed.

Conclusions: The present shunt-related model successfully simulated a hypertensive status in pulmonary circulation and reproduced the characteristic transition of pulmonary vasculopathy from reversibility to irreversibility within a relatively short period. Thus, this model could offer an alternative with low mortality and high reproducibility for investigations on the underling mechanisms of shunt-related pulmonary hypertension. ( $\mathrm{J}$ Thorac Cardiovasc Surg 2013;146:1220-6)

Supplemental material is available online.

In patients with systemic-to-pulmonary shunts, the status of the pulmonary vascular structure and the extent of pulmonary hypertension $(\mathrm{PH})$ are important determinants of the feasibility of corrective procedures, the clinical course, and long-term survival. Recently, drugs targeting the signaling pathways involved in the pathogenesis of $\mathrm{PH}$ have been confirmed to partially or completely reverse the hypoxia/

From the State Key Laboratory of Cardiovascular Disease, ${ }^{\mathrm{a}}$ and Department of Surgery, ${ }^{\mathrm{b}}$ National Center for Cardiovascular Disease, Fuwai Hospital, Chinese Academy of Medical Sciences and Peking Union Medical College, Beijing, People's Republic of China.

This work was supported by research grants from the National 973 Program of China (grant 2010CB529505 to SS.H.).

Disclosures: Authors have nothing to disclose with regard to commercial support. LK.M. and XY.L. contributed equally to this work.

Received for publication Sept 29, 2012; revisions received Dec 9, 2012; accepted for publication Jan 11, 2013; available ahead of print Feb 11, 2013.

Address for reprints: Yingjie Wei, PhD, or Shengshou Hu, MD, 167 Beilishi Rd, Beijing 100037, People's Republic of China (E-mail: hushengshou@yahoo.com). $0022-5223 / \$ 36.00$

Copyright (C) 2013 by The American Association for Thoracic Surgery

http://dx.doi.org/10.1016/j.jtcvs.2013.01.018 monocrotaline-induced PH in rats. However, these targeted drugs only exhibited partial improvements in symptoms, exercise tolerance, and hemodynamic indexes, with slight efficacy on mortality in patients with severe $\mathrm{PH} .{ }^{1}$ This has largely attenuated the dogma that the hypoxia/ monocrotaline-induced PH model should be used to delineate the potential mechanism of $\mathrm{PH}$, including shuntrelated $\mathrm{PH}^{1-3}$ and thus would be the most scientific method for determining the underlying mechanism of hyperkinetic $\mathrm{PH}$ in experimentally validated shunt-related models.

Many shunt-related models have been described and studied. These have included peripheral shunts established in the cervical, ${ }^{4}$ abdominal, ${ }^{5}$ or femoral ${ }^{6}$ unit and central shunts prepared between the left innominate artery, ${ }^{7}$ left carotid artery, ${ }^{8}$ or ascending aorta ${ }^{9}$ and pulmonary artery trunk. However, peripheral shunts have only induced limited pulmonary vascular remodeling (reversible grade 1). The high-level surgical requirements, relatively greater mortality, ${ }^{10}$ and inadequacy of biochemical reagents for large animals have impeded the adoption of central shunt models; thus, an acceptable shunt-related model is still lacking.

Therefore, we created an original, reproducible, shuntrelated model in rats using a 2 -stage surgical procedure. 


$$
\begin{aligned}
& \text { Abbreviations and Acronyms } \\
& \begin{aligned}
\text { CSG } & =\text { combined surgery group } \\
\text { LCCA } & =\text { left common carotid artery } \\
\text { LCSG } & =\text { left cervical shunt group } \\
\text { LEJV } & =\text { left external jugular vein } \\
\text { mPAP } & =\text { mean pulmonary arterial pressure } \\
\text { MT } & =\text { medial thickness } \\
\text { PASP } & =\text { pulmonary arterial systolic pressure } \\
\text { PH } & =\text { pulmonary hypertension } \\
\text { RPALG } & =\text { right pulmonary artery ligation group } \\
\text { RVHI } & =\text { right ventricular hypertrophy index } \\
\text { RVSP } & =\text { right ventricular systolic pressure } \\
\text { SD } & =\text { Sprague-Dawley } \\
\text { SOG } & =\text { sham operation group }
\end{aligned}
\end{aligned}
$$

Subsequently, we performed hemodynamics evaluation, morphologic analysis, and blood gas analysis to explore the extent of PH achieved with this model.

\section{METHODS}

Male Sprague-Dawley (SD) rats from Vital River Laboratory Animal (Beijing, China) were used (age, 8 weeks; weight, $280 \pm 20$ g). They were randomly allocated to the right pulmonary artery ligation group (RPALG, $n=16$ ), left cervical shunt group (LCSG, $n=16$ ), combined surgery group (CSG, $n=32$ ), and sham operation group (SOG, $n=24$ ).

All rats received humane care in compliance with the "Guide for the Care and Use of Laboratory Animals" (National Institutes of Health publication no. 85-23, National Academy Press, Washington, DC, revised 1996). The ethics committee on animal study of Fuwai Hospital approved the experimental protocol.

\section{Discrepancy in Bilateral Lungs of SD Rats}

The right lung of SD rats consists of 4 lobes and the left lung of only 1 lobe. Although this unique arrangement has been attributed to the anatomic deflection of the heart, we still needed to ascertain its true implications in creating shunt-related $\mathrm{PH}$.

Bilateral lungs of age-, gender-, and weight-matched SD rats $(\mathrm{n}=36)$ were collected and individually weighed before and after oven drying at $50^{\circ}$ for 72 hours. Next, the relationship between the bilateral lungs was assessed by plotting the wet or dry weight of the right lung to that of the left lung (Figure 1). The mean wet ratio was $1.96 \pm 0.11$ and the mean dry weight ratio was $1.95 \pm 0.11$. Using this anatomic discrepancy and the extant surgical procedure (ligation of the pulmonary artery), ${ }^{11}$ we ligated the right pulmonary artery to increase the pulmonary blood flow in the left lung.

\section{Surgical Protocol}

The rats were anesthetized with $10 \%$ chloral hydrate and atropine sulfate $(3 \mathrm{~mL} / \mathrm{kg}$ and $0.1 \mathrm{mg}$, respectively, intraperitoneally), orotracheally intubated, and immobilized in a left lateral decubitus position. Ventilation was maintained with a Harvard Rodent Ventilator (Inspira ASVP, Harvard Apparatus, Holliston, Mass) for the whole procedure.

\section{Right Pulmonary Artery Ligation Group}

Under sterile conditions, the right pulmonary artery was approached through a posterolateral thoracotomy in the third intercostal space and then ligated (10-0 nylon suture) after dissecting the interspace between right superior vena cava and right primary bronchus (Figure 2, A). Next, the chest was closed in layers (3-0 silk suture), and all the air in the right pleural space was evacuated through a tube of polyethylene 50 , serving as a chest drainage tube. Finally, the rats were weaned from mechanical ventilation and allowed to recover with continuous supplemental oxygen. However, the endotracheal tube was not extubated until their respiration had stabilized.

\section{Left Cervical Shunt Group}

Anesthesia was induced as described for the previous group, and systemic heparinization was implemented by heparin sodium (100 IU, intraperitoneally). During spontaneous breathing, the rats were fixed in a supine position, and the left common carotid artery (LCCA) and left external jugular vein (LEJV) were exposed. Next, the proximal LCCA was clamped with microvascular clips and the distal portion was ligated at the bifurcation point. The proximal LEJV was suspended to the adjacent jugular muscle by microscopic suture, and the distal portion was ligated at the confluence site. Subsequently, the LCCA was amputated before the bifurcation and immediately flushed with heparin $(50 \mathrm{U} / \mathrm{mL})$. Next, the proximal stump was brought through and everted over a 2-mm-long, 18-gauge, intravenous catheter and circumferentially ligated in the middle of the cuff body. Then, the proximal stump of the LCCA was rotated and inserted into the LEJV lumen through a transverse incision in the anterior wall of the LEJV and the anastomosis was secured by ligatures (Figure 2, $B$ ). Finally, the suspended suture and the vascular clips were sequentially removed, and the patency of the shunt was confirmed visually by the presence of a continuous thrill, apparent dilation, and contradictory color change of the proximal part of the LEJV.

\section{Combined Surgery Group}

The combined surgery was performed as a 2-stage procedure. In brief, the right pulmonary artery was ligated, and, 1 week later, the left cervical shunt was established (Figure 2).

\section{Sham Operation Group}

The rats in the SOG underwent the 2-stage procedure, except for ligation of the right pulmonary artery and generation of the cervical communication.

\section{Postoperative Care}

Postoperatively, the rats were kept in a constant-temperature room $\left(21^{\circ} \mathrm{C}\right.$; relative humidity, $\left.50 \%-70 \%\right)$, exposed to a normal light/dark cycle, and provided standard laboratory chow and water ad libitum. Aspirin (75 $\mathrm{mg} / \mathrm{kg} / \mathrm{d})$ and hydrochlorothiazide $(25 \mathrm{mg} / \mathrm{d})$ were administered by hydroposia to prevent anastomosis occlusion and lung edema.

\section{Hemodynamics}

The pulmonary pressure changes were measured in the closed-chest rats with a technique routinely used in our laboratory. ${ }^{12}$ In brief, a 13-cm-long, heparin-priming polyethylene catheter (outer diameter, $0.9 \mathrm{~mm}$ ) connected to PowerLab 16/30 (ADInstruments, Dunedin, New Zealand) through a pressure transducer was introduced into the right external jugular vein and advanced into the right ventricle and the main pulmonary artery. The right ventricular systolic pressure (RVSP), pulmonary arterial systolic pressure (PASP), and mean pulmonary arterial pressure (mPAP) were recorded.

Immediate effect test. The acute effects of the 4 different surgical interventions on the pulmonary circulation were evaluated immediately after surgery. The shunt clamping test was performed to evaluate the reversibility of PH in the LCSG, CSG, and SOG.

Chronic effect test. The chronic effects of the 4 different surgical procedures on the pulmonary vasculature were investigated at 8 and 12 weeks after the surgical intervention. The baseline data of the hemodynamic variables before and after occlusion of the cervical shunt was measured in the LCSG, CSG, and SOG. 


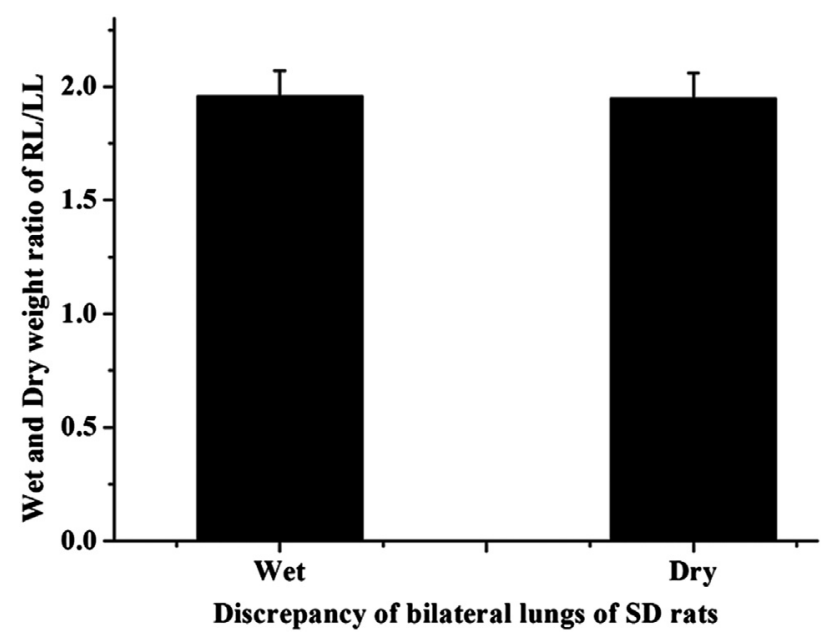

FIGURE 1. Wet and dry weight ratio of right lung $(R L) /$ left lung $(L L)$ of age-, gender-, and weight-matched Sprague-Dawley $(S D)$ rats. Mean wet weight ratio was $1.96 \pm 0.11$ and dry weight ratio was $1.95 \pm 0.11$, indicating RL had more flow reversal than LL.

\section{Blood Gas Analysis}

A blood sample $(0.3 \mathrm{~mL})$ was taken from the femoral artery and analyzed in a blood gas analyzer (Nova Biomedical, pHOx Ultra, Waltham Mass). The partial arterial pressure of oxygen, blood oxygen saturation, hemoglobin concentration, and hematocrit were determined.

\section{Organ Harvesting}

After the hemodynamic measurements, the left lung was perfused with $4^{\circ}$ phosphate-buffered saline through the inferior cava vena. Next, the heart-lung tissues were immediately extirpated en bloc. The lower part of the left lung was rinsed in $10 \%$ neutral-buffered formalin through the left principal bronchus under constant pressure until fully inflated and immersed in $10 \%$ formalin for 24 hours at room temperature. Then, the fixed tissues were trimmed to an appropriate size, placed in embedding cassettes, dehydrated in graded ethanol dilutions, cleared in xylene solution, and embedded in paraffin.

\section{Morphologic Analysis}

Four $4-\mu \mathrm{m}$-thick sections were cut from each paraffin block. Two were stained with hematoxylin-eosin for routine light microscopic examination, and two were stained with modified Weigert's method to demonstrate the elastic tissue and counterstained with Van Gieson's solution to show the muscle and collagen. All the small muscular pulmonary arteries discriminated by the accompanying airways were systematically scanned for graded lesions in the pulmonary arteries and arterioles according to the Heath-Edwards classification. ${ }^{13}$ The presence of medial hypertrophy was carefully graded according to well-established quantitative protocols ${ }^{9}$ using an Olympus imaging analyzer system (Olympus BX61, Tokyo, Japan). The medial thickness (MT) was determined as the mean distance between the internal and external elastic lamina, and the percentage of MT was calculated using the following formula: percentage of MT $=2 \times$ MT/external diameter $\times 100 \%$.

The pulmonary vasculopathy evaluation was performed by 1 of us (J.M.), who did not know the source of the slides. The reversible and irreversible lesions were demarcated according to the standards presented by Heath and Edwards, ${ }^{13}$ Wagenvoort, ${ }^{14}$ and Wagenvoort and colleauges. ${ }^{15}$ Muscularization (grade 1) and cellular intimal proliferation (grade 2) were judged as reversible lesions, and intimal fibrosis and entire luminal occlusion (grade 3) were classified as irreversible lesions.

\section{Right Ventricular Hypertrophy Evaluation}

The free wall of the right ventricle was separated from the ventricular septum. Next, the right and left ventricles plus the septum were blotted and weighed. Finally, the right ventricular hypertrophy index (RVHI) was calculated as the ratio of the weight of right ventricle to that of the left ventricle plus the septum. ${ }^{8}$

\section{Statistical Analysis}

The data are presented as the mean \pm standard deviation. The data from the rats in the CSG were compared with those from the rats in the RPALG, LCSG, and SOG using SPSS, version 13.0, statistical software (SPSS, Chicago, Ill). The data from the rats in the CSG and RPALG, LCSG, and SOG were compared using independent-samples $t$ tests. The data from the same group before and after closure of the cervical shunt were compared using paired-samples $t$ tests.

\section{RESULTS \\ Survival}

All the rats survived the operation. Three postoperative deaths occurred in the CSG, one in the SOG, and none in the RPALG and LCSG. A postmortem examination was performed to ascertain the cause of death. The 3 deaths in the CSG were from postoperative right heart failure, and
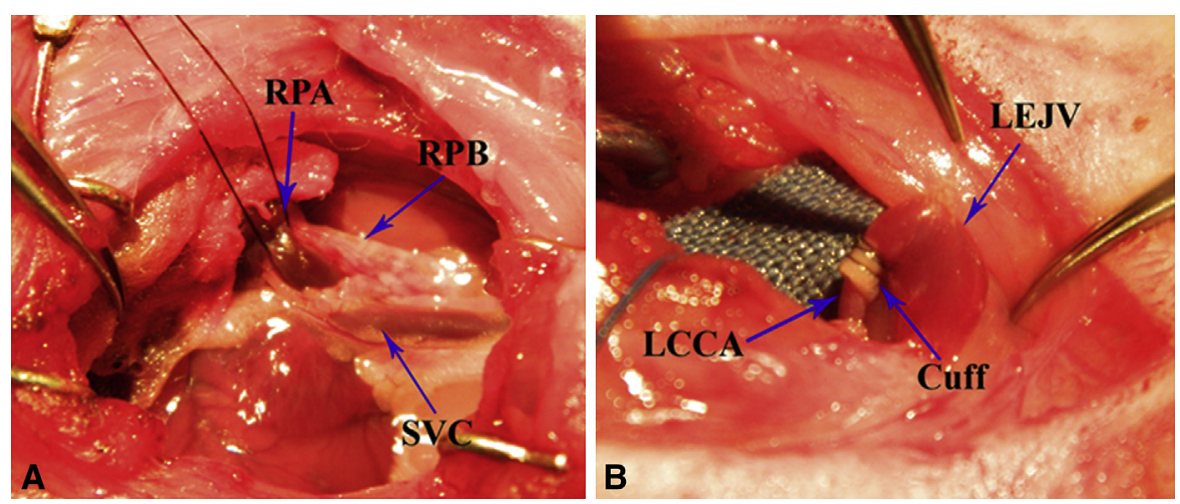

FIGURE 2. A, Intraoperative photograph of first-stage procedure-ligation of right pulmonary artery (RPA). After posterolateral thoracotomy in third intercostal space, the RPA was exposed and ligated after mobilizing interspace between right superior vena cava (SVC) and right primary bronchus $(R P B)$. B, Second-stage surgery - cervical shunt communication procedure. Cervical shunt between left common carotid artery (LCCA) and left external jugular vein $(L E J V)$ was established using a cuff technique. 

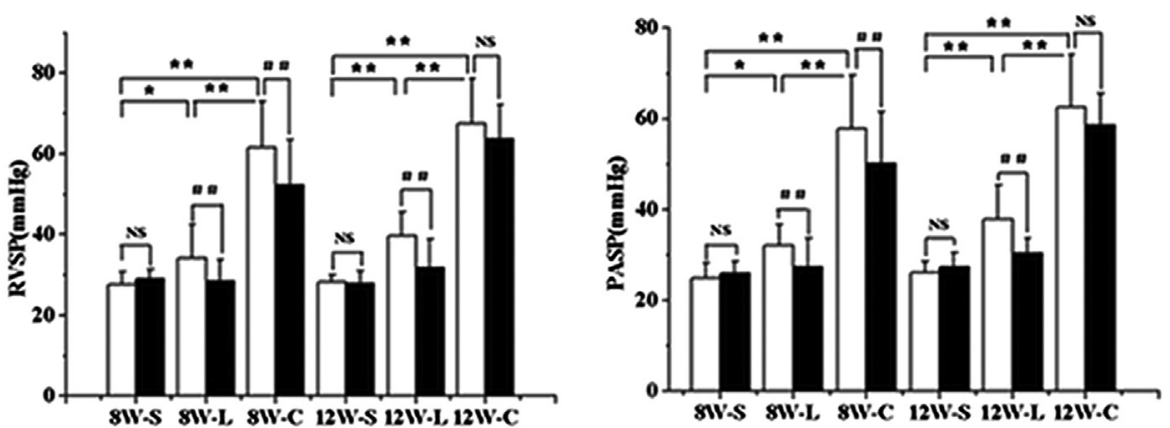

A

B
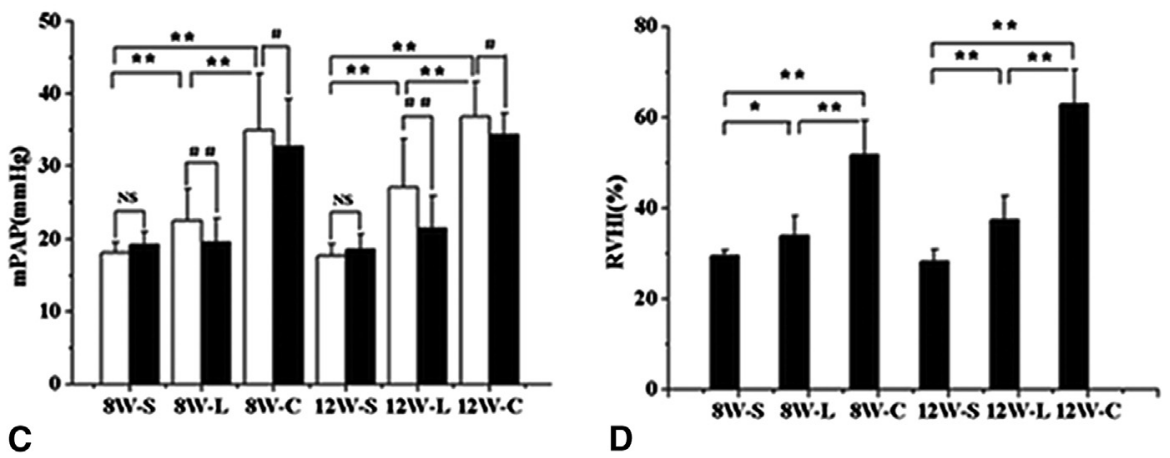

FIGURE 3. A, Right ventricular systolic pressure (RVSP) changes before (white bar) and after (solid bar) closure of cervical shunt. Mean values for RVSP showed time-related increment in left cervical shunt group $(L C S G)$ and combined surgery group $(C S G)$, with significant differences between CSG and LCSG and sham operation group (SOG) at 8 and 12 weeks postoperatively. Shunt clamping induced significant decreases in RVSP in LCSG at 8 weeks postoperatively $(8 W-L)$, CSG at 8 weeks postoperatively $(8 W-C)$, and LCSG at 12 weeks postoperatively $(12 W-L)$ but not in CSG at 12 weeks postoperatively $(12 W$ $C)$. No significant differences were found between right pulmonary artery ligation group (RPALG) and LCSG $(P>.50)$; thus, only RVSP in LCSG is shown. $\mathrm{B}$, Pulmonary arterial systolic pressure $(P A S P$ ) changes before (open bar) and after (solid bar) closure of cervical shunt. Mean values for PASP showed timerelated increment in LCSG and CSG, with significant differences between CSG and LCSG and SOG at 8 and 12 weeks postoperatively. Shunt clamping induced significant decreases in PASP in $8 \mathrm{~W}-\mathrm{L}, 8 \mathrm{~W}-\mathrm{C}$, and $12 \mathrm{~W}-\mathrm{L}$ but not in $12 \mathrm{~W}-\mathrm{C}$. No significant differences were found between RPALG and LCSG $(P>.50)$; thus, only PASP in LCSG is shown. C, Mean pulmonary arterial pressure ( $m P A P)$ changes before (open bar) and after (solid bar) closure of cervical shunt. Mean mPAPs showed time-related increment in LCSG and CSG, with significant differences between CSG and LCSG and SOG at 8 and 12 weeks. Shunt clamping induced significant decreases in mPAP of CSG and LCSG at 8 and 12 weeks. No significant differences were found between RPALG and LCSG $(P>.50)$; thus, only mPAP in LCSG is shown. D, Right ventricle/left ventricle plus septum weight ratio changes in RPALG, LCSG, CSG, and SOG. Right ventricular hypertrophy index $(R V H I)$ in LCSG and CSG showed time-related increments, with significant differences in RVHI between CSG and LCSG and SOG at 8 and 12 weeks. No significant differences were found between RPALG and LCSG $(P>.50)$; thus, only mPAP in LCSG is shown. Data presented as mean \pm standard deviation. ${ }^{*} * P<.01, * P<.05$, \#\#P<.01,\#P<.05, and ${ }^{\mathrm{NS}} P>.05$. $8 W$-S, SOG at 8 weeks postoperatively; $8 W-C, \mathrm{CSG}$ at 8 weeks postoperatively; $12 \mathrm{~W}$-S, SOG at 12 weeks postoperatively.

the 1 death in the SOG was from unknown causes. The remaining rats survived and were all included in the final data analysis. The total survival rate was $90.63 \%$ in the CSG. Three rats in the CSG ( 1 at 8 weeks and 2 at 12 weeks) died during right heart catheterization. Of the 3 rats, 2 died of cardiac tamponade because the catheter tip penetrated the right atrium wall and protruded into the pericardial cavity. However, the third died of unknown reasons. Therefore, the hemodynamic indexes were not obtained from these animals. Otherwise, slow weight gain was observed with high frequency in the CSG (Table E1).

\section{Anastomosis Patency Status}

At the harvesting points, most rats in the CSG and LCSG demonstrated conspicuous pulsation and a vigorous thrill in the left supraclavicular fossa. However, in 2 rats in the CSG, no evident thrill was observed at 12 weeks, indicating the presence of systemic levels of PH. The patency was also examined by direct vision of the anastomosis lumen. Despite overt intimal hyperplasia in the LEJV in sporadic cases, the cervical shunts were all still patent.

\section{Blood Gas Analysis}

Compared with the RPALG, LCSG, and SOG, a significant decrement in the partial arterial pressure of oxygen and a slight increment in the hemoglobin and hematocrit were progressively present in the CSG as the disorder advanced (Table E1).

\section{In Vivo Hemodynamic Evaluation}

Immediate effect. Immediately after cervical surgery, the RVSP, PASP, and mPAP in the CSG were dramatically 
elevated. Occlusion of the shunt induced an instant decrease in the RVSP, PASP, and mPAP, although these were still greater than the corresponding normal levels (Figure 3 and Table E2). This finding could be interpreted as the combined surgery resulting in a prompt, persistent, and reversible increment in pulmonary arterial pressure.

Chronic effect. As demonstrated in Figure 3, A-C, and Table E2, the mean values for the RVSP, PASP, and mPAP of the CSG manifested a prominent and consistent timerelated increment compared with the remaining groups. Completely clamping the shunt in the CSG induced a significant decrease in the RVSP, PASP, and mPAP at 8 weeks, although the levels remained much greater than those of the SOG. Although a reduction in cardiac output from closing the shunt might play a role in the decrease, considering the pulmonary vasculopathy at 8 weeks in the CSG (Figure 5), the PH status still possessed a reversible characteristic at 8 weeks postoperatively. Cervical shunt occlusion also induced a reduction in these hemodynamic variables at 12 weeks in the CSG, but most of these decreases failed to reach a statistically significant difference, confirming an approximate demarcation of the reversibility of the gradual remodeling of the pulmonary vasculature.

However, completely clamping the cervical shunt in the LCSG resulted in regression of the hemodynamic indexes to the normal levels of the SOG at 12 weeks (Figure 3, A$C$ ), suggesting that the pulmonary vasculature was still in a reversible condition.

\section{Right Ventricular Hypertrophy Index}

RVHI increased in a time-related manner in the RPALG, LCSG, and CSG compared with that of the SOG (Table E2 and Figure 3,D). Although the right ventricular hypertrophy in the CSG was more severe than that in the RPALG and LCSG and a significant difference in the RVHI was present between the CSG and the RPALG and LCSG, no statistically significant difference was found between the RPALG and LCSG in the RVHI.

\section{Morphologic Analysis of Pulmonary Vasculature}

As demonstrated in Figure 4, the pulmonary vasculature in the CSG was extensively remodeled at the levels of the arteries less than $125 \mu \mathrm{m}$ in external diameter compared with the pulmonary vasculature of the SOG. Histopathologic analysis revealed significant medial hypertrophy in the intra-acinar arteries 25 to $75 \mu \mathrm{m}$ in external diameter and a pervasive recurrence of the smooth muscle layer in the peripheral arterioles less than $25 \mu \mathrm{m}$ in external diameter in the CSG. Moreover, compared with the SOG, the vessel muscularization in the CSG was progressively exacerbated in terms of the number of vessels having a full or partial muscular wall at all levels.

Furthermore, a striking spectrum of pulmonary remodeling lesions gradually presented with the deterioration of

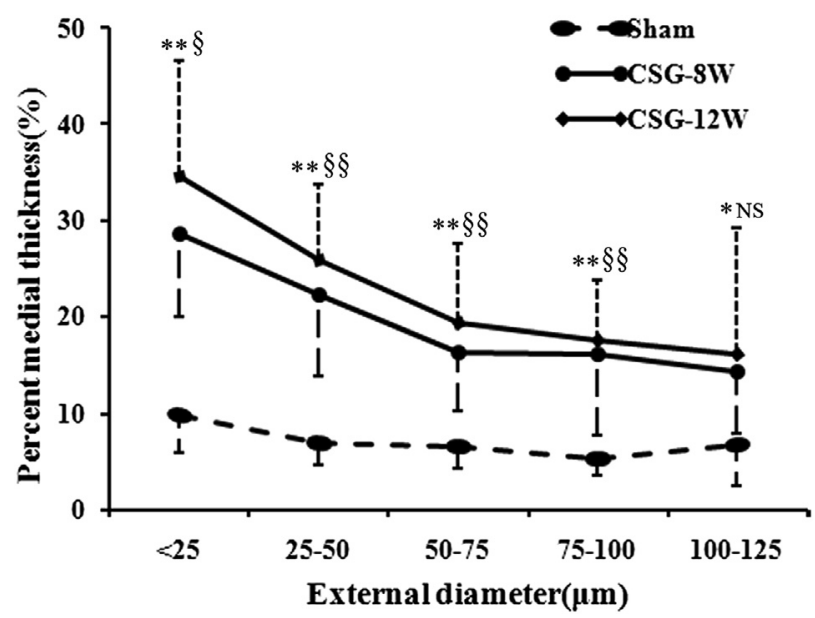

FIGURE 4. Plot showing rats in combined surgery group (CSG) have increased medial thickness of arteries or arterioles less than $125 \mu \mathrm{m}$ in external diameter. Histopathologic analysis revealed significant medial hypertrophy in intra-acinar arteries of 25 to $75 \mu \mathrm{m}$ in external diameter and pervasive recurrence of smooth muscle layer in peripheral arterioles less than $25 \mu \mathrm{m}$ in external diameter in CSG. Moreover, compared to 8-week value, extent of right ventricular hypertrophy index $(R V H I)$ in CSG at 12 weeks was aggravated in arteries or arterioles less than $125 \mu \mathrm{m}$ in external diameter. Data presented as mean \pm standard deviation. $* P<.05$ versus sham operation group (SOG), $* * P<.01$ versus SOG, $\S P<.05$ versus CSG at 8 weeks postoperatively, $\S \S P<.01$ versus CSG at 8 weeks postoperatively, ${ }^{\mathrm{NS}} P>.05$ versus $\mathrm{CSG}$ at 8 weeks postoperatively. $C S G-8 W, \mathrm{CSG}$ at 8 weeks postoperatively; CSG-12W, CSG at 12 weeks postoperatively.

this disorder in the CSG. The predominant reversible changes-muscularization and cellular intimal proliferation-had emerged by 8 weeks postoperatively and were progressively exacerbated into irreversible vascular lesions-intimal fibrosis and entire luminal occlusion-by 12 weeks postoperatively. Representative images from the CSG and SOG are shown in Figure 5.

Consistent with the hemodynamic data, no apparent vascular remodeling was observed in the SOG, although medial hypertrophy was present in the small pulmonary arteries in the RPALG and LCSG at 8 weeks postoperatively. However, this slight vascular remodeling lesion was not aggravated at 12 weeks in terms of the percentage of MT and also did not extend into the peripheral arterioles.

\section{DISCUSSION}

In the present study, we successfully established a novel rat model of $\mathrm{PH}$ to closely reproduce the aberrant hemodynamic status and characteristic morphologic changes observed in the lungs of patients with intracardiac shunts. Congenital intracardiac shunts consequently result in $\mathrm{PH}$ and, eventually, Eisenmenger syndrome, if left without surgical correction. In some cases, even with surgical correction, the exaggerated pulmonary vascular vasospasm frequently provokes postoperative pulmonary hypertensive crisis, which inevitably increases mortality. Although the 


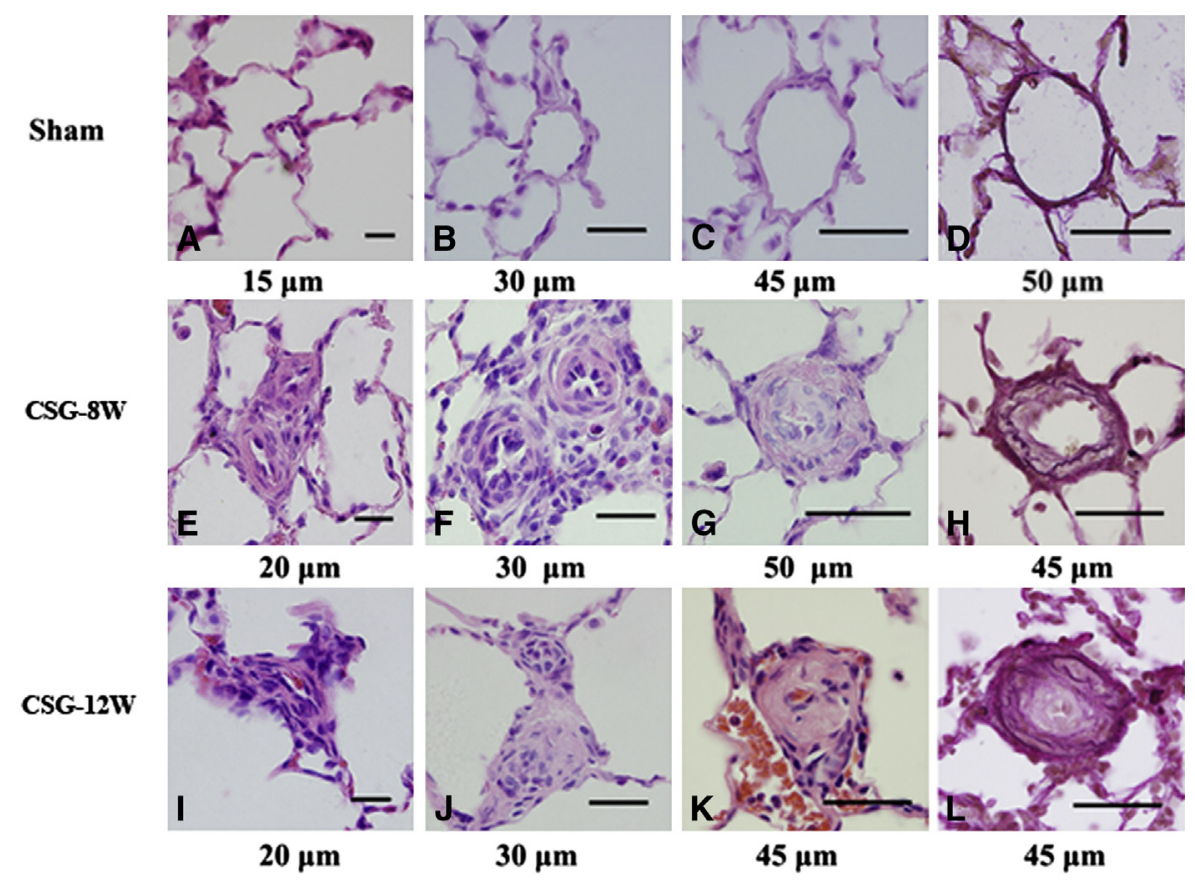

FIGURE 5. Representative morphologic images of lungs from sham operation group ( $S O G)$ and combined surgery group (CSG). A-D, Pulmonary arteries and arterioles less than $50 \mu \mathrm{m}$ from SOG with normal morphology. Pulmonary arteries and arterioles less than $50 \mu \mathrm{m}$ from CSG at 8 weeks with E, muscularization, F, cellular intimal proliferation, and G, H, neointima formation. Pulmonary arteries and arterioles less than $50 \mu \mathrm{m}$ from CSG at 12 weeks showing I, muscularization, J, cellular vascular occlusion, and $\mathrm{K}$, intimal fibrosis and L, entire luminal occlusion. CSG-8W, CSG at 8 weeks postoperatively; $C S G-12 W$, CSG at 12 weeks postoperatively.

characteristically pathologic lesions in the pulmonary vessels have been previously described in detail, ${ }^{5}$ the progression of reversible lesions to irreversible lesions has never been successfully reproduced in rats with left-to-right shunts.

The classic hypoxia/monocrotaline-induced PH models have deduced numerous cellular and molecular mechanisms of PH. However, their easy and reproducible merits would not negate that the hypoxia/monocrotaline-induced $\mathrm{PH}$ has been attributed, to a large extent, to the sustained vasoconstriction. ${ }^{15-17}$ In particular, no occlusive intimal lesions emerged in the peripheral pulmonary arteries/arterioles, let alone the loss of pulmonary microvessels. ${ }^{17-19}$ Furthermore, recent clinical trials ${ }^{1}$ have shown that monotherapy or combination therapy using targeted drugs exerted preventive, or even reversible, efficacy on hypoxia/monocrotaline-induced $\mathrm{PH}^{20,21}$ However, none resulted in significantly lower mortality in patients with severe PH. This disappointing discrepancy has meant that researchers must reconsider the incompetence and limitations of hypoxia/monocrotaline-induced $\mathrm{PH}^{22-24}$ Also, investigators evaluating new therapies for $\mathrm{PH}$ should adopt an alternative PH model.

Continuous attempts have been made to simulate the pulmonary vasculopathy by surgically creating peripheral or central systemic-to-pulmonary communication. ${ }^{4-8,10}$ Although the pulmonary circulation is relatively well tolerant to peripheral shunts, ${ }^{4-6}$ the limited increase in pulmonary blood flow unavoidably results in too little or only medial hypertrophy changes in the pulmonary arteries (grade 1). Central shunts prepared in large animals $^{7,8,10}$ closely simulate the pathophysiologic characteristics of congenital intracardiac shunts; however, the high-level surgical requirements, relatively greater mortality, ${ }^{10}$ limited potency in inducing remodeling lesions, ${ }^{7}$ and inadequacy of biochemical reagents for large animals have impeded the widespread adoption of these models. Thus, a satisfactory shunt-related model that could be used to determine the underlying molecular mechanisms of pulmonary vascular remodeling due to in vivo exposure to high blood flow is still needed. Therefore, it is critically important to create an experimentally validated shunt model.

In the present study, we first found the anatomic discrepancy between the bilateral lungs in the SD rats, which provided an advantage in generating the shunt model. With this finding and 2 extant surgical procedures used in creating flow-associated PH (ie, cervical shunt ${ }^{4}$ and pulmonary artery ligation ${ }^{11}$ ), we created an original rat model of high kinetic unilateral PH. Although 1 lung was excluded from the pulmonary circulation, and this could generate a ventilation/perfusion mismatch, as demonstrated by the blood gas analysis results in the RPALG (Table E1), the remaining left lung was relatively tolerant to the increased pulmonary blood flow and could maintain a satisfactory balance between oxygen supply and expenditure. 
As presented in Figure 3, compared with the LCSG and RPALG, this 2-stage procedure did result in striking elevations of the RVSP, PSAP, mPAP, and RVHI, with surprisingly low mortality in the CSG. Furthermore, these hemodynamic changes were partially reversible immediately and 8 weeks after the 2-stage surgery and gradually progressed to irreversible lesions by 12 weeks postoperatively. Consistent with the hemodynamic changes, after the cervical surgery in the CSG, reversible changes in the pulmonary vasculature (ie, medial hypertrophy and cellular intimal reaction) had occurred at 8 weeks and gradually progressed into boundary lesions (excessively cellular intimal proliferation) and finally to irreversibly acellular intimal fibrosis and total luminal occlusion by 12 weeks (Figure 5). Taken together, we can conclude that the pulmonary vascular remodeling induced by this original model experienced a transition from reversibility at 8 weeks to irreversibility by 12 weeks.

Some limitations of the present study should be clarified. The cuff technique used in the cervical procedure might disallow for shunt flow to progressively and concomitantly augment in growing rats. Thus, the true potential of this model and the extent of PH might have been inadvertently underestimated. This could be another explanation for the absent concentric laminar intimal fibrosis and plexiform lesions, except for extrapolating from the relatively short observation time. No long-term analysis after shunt closure was performed to observe the effect on the pulmonary vasculopathy.

\section{CONCLUSIONS}

We have presented an original unilateral $\mathrm{PH}$ model in rats based on their inherent discrepancy between the bilateral lungs. This model provides an alternative for investigations directed at elucidating the underlying mechanisms responsible for pulmonary vasculopathy, especially the switch from reversibility to irreversibility.

Special gratitude should be given to Professor Yue Tang, Peihe Wang, Liujun Jia, and Fuliang Luo for their technical assistance.

\section{References}

1. Bai Y, Sun L, Hu S, Wei Y. Combination therapy in pulmonary arterial hypertension: a meta-analysis. Cardiology. 2011;120:157-65.

2. Humbert M, Morrell NW, Archer SL, Stenmark KR, MacLean MR, Lang IM, et al. Cellular and molecular pathobiology of pulmonary arterial hypertension. J Am Coll Cardiol. 2004;43(12 suppl S):13S-24S.

3. Voelkel NF, Tuder RM, Weir EK. Pathophysiology of primary pulmonary hypertension. In: Rubin L, Rich S, eds. Primary pulmonary hypertension. New York: Marcel Dekker; 1997:83-129.
4. Xiong M, Yao JP, Wu ZK, Liao B, Liang YJ, Zhang X, et al. Fibrosis of pulmonary vascular remodeling in carotid artery-jugular vein shunt pulmonary artery hypertension model of rats. Eur J Cardiothoracic Surg. 2012;41:162-6.

5. Li XH, Jin HF, Bin G, Wang L, Tang C, Du J. Endogenous hydrogen sulfide regulates pulmonary artery collagen remodeling in rats with high pulmonary blood flow. Exp Biol Med. 2009;234:504-12.

6. Fullerton DA, Mitchell MB, Jone DN, Maki A, McIntyre RC Jr. Pulmonary vasomotor dysfunction is produced with chronically high pulmonary blood flow. J Thorac Cardiovasc Surg. 1996;111:190-7.

7. Rondelet B, Dewachter C, Kerbaul F, Kang X, Fesler P, Brimioulle S, et al. Prolonged overcirculation-induced pulmonary arterial hypertension as a cause of right ventricular failure. Eur Heart J. 2012;33:1017-26.

8. Wang W, Liu R, Cao G, Zhang F, Zhang Y, Zhang Z, et al. A reliable rabbit model for hyperkinetic pulmonary hypertension. J Thorac Cardiovasc Surg. 2010;140: 395-9.

9. Rabinovitch M, Haworth SG, Castaneda AR, Nadas AS, Reid LM. Lung biopsy in congenital heart disease: a morphometric approach to vascular disease. Circulation. 1978;58:1107-22.

10. Reddy VM, Meyrick B, Wong J, Khoor A, Liddicoat JR, Hanley FL, et al. In utero placement of aorta pulmonary shunts: a model of postnatal pulmonary hypertension with increased pulmonary blood flow in lambs. Circulation. 1995;92:606-13.

11. Gorenflo M, Herpel E, Ullmann MV, Rohlig K, Demirakca S, Klimpel H, et al. Pulmonary vascular changes in piglets with increased pulmonary blood flow and pressure. Virchow Arch. 2007;450:643-52.

12. Sun P, Liu WL. Method for measuring pulmonary artery pressure by right cardiac catheter in rats. Acta Acad Med Sin. 1984;6:465-7.

13. Heath D, Edwards JE. The pathology of hypertensive pulmonary vascular disease: a description of six grades of structural changes in the pulmonary arteries with special reference to congenital cardiac septal defects. Circulation. 1958; 18:533-47

14. Wagenvoort CA. Open lung biopsies in congenital heart disease for evaluation of pulmonary vascular disease: predictive value with regard to corrective operability. Histopathology. 1985;9:417-36.

15. Wagenvoort CA, Wagenvoort N, Draulans-Noe Y. Reversibility of plexogenic pulmonary arteriopathy following banding of the pulmonary artery. $J$ Thorac Cardiovasc Surg. 1984;87:876-86.

16. Oka M, Fagan KA, Jones PL, McMurtry IF. Therapeutic potential of rhoa/rho kinase inhibitors in pulmonary hypertension. Br J Pharmacol. 2008;155:444-54.

17. Tenmark KR, McMurtry IF. Vascular remodeling versus vasoconstriction in chronic hypoxic pulmonary hypertension: a time for reappraisal? Circ Res. 2005;97:95-8.

18. McLoughlin P, McMurtry I. Counterpoint: chronic hypoxia-induced pulmonary hypertension does not lead to loss of pulmonary vasculature. J Appl Physiol. 2007;103:1451-3; discussion 1453-4.

19. Rabinovitch M, Chesler N, Molthen RC. Point: counterpoint: chronic hypoxiainduced pulmonary hypertension does/does not lead to loss of pulmonary vasculature. J Appl Physiol. 2007;103:1449-51.

20. Itoh T, Nagaya N, Fujii T, Iwase T, Nakanishi N, Hamada K, et al. A combination of oral sildenafil and beraprost ameliorates pulmonary hypertension in rats. Am J Respir Crit Care Med. 2004;169:34-8.

21. Eddahibi S, Hanoun N, Lanfumey L, Lesch KP, Raffestin B, Hamon M, et al. Attenuated hypoxic pulmonary hypertension in mice lacking the 5-hydroxytryptamine transporter gene. J Clin Invest. 2000;105:1555-62.

22. Bauer NR, Moore TM, McMurtry IF. Rodent models of PAH: are we there yet? Am J Physiol Lung Cell Mol Physiol. 2007;293:L580-2.

23. Voelkel NF, Tuder RM. Hypoxia-induced pulmonary vascular remodeling: a model for what human disease? J Clin Invest. 2000;106:733-8.

24. Zaiman A, Fijalkowska I, Hassoun PM, Tuder RM. One hundred years of research in the pathogenesis of pulmonary hypertension. Am J Respir Cell Mol Biol. 2005;33:425-31. 
TABLE E1. Weight gain and oxygenation indexes

\begin{tabular}{|c|c|c|c|c|c|}
\hline \multirow[b]{2}{*}{ Variable } & \multirow[b]{2}{*}{ Weight gain (g) } & \multicolumn{4}{|c|}{ Arterial blood gas parameters } \\
\hline & & $\mathrm{PaO}_{2}(\mathrm{~mm} \mathrm{Hg})$ & $\mathrm{SaO}_{2}(\%)$ & $\mathbf{H b}(\mathrm{g} / \mathrm{dL})$ & Het $(\%)$ \\
\hline \multicolumn{6}{|l|}{ RPALG } \\
\hline $8 \mathrm{~W}(\mathrm{n}=8)$ & $527.43 \pm 33.71$ & $95.27 \pm 3.49$ & $97.04 \pm 2.25$ & $13.59 \pm 1.36$ & $42.30 \pm 2.39$ \\
\hline $12 \mathrm{~W}(\mathrm{n}=8)$ & $570.33 \pm 25.84$ & $92.08 \pm 4.63$ & $94.34 \pm 3.38$ & $15.16 \pm 0.93$ & $45.37 \pm 1.80$ \\
\hline \multicolumn{6}{|l|}{ LCSG } \\
\hline $8 \mathrm{~W}(\mathrm{n}=8)$ & $537.02 \pm 26.39$ & $101.34 \pm 5.09$ & $98.17 \pm 1.25$ & $12.81 \pm 2.03$ & $45.83 \pm 1.71$ \\
\hline $12 \mathrm{~W}(\mathrm{n}=8)$ & $589.48 \pm 35.01$ & $98.08 \pm 3.72$ & $97.39 \pm 1.83$ & $13.29 \pm 1.27$ & $43.04 \pm 1.94$ \\
\hline \multicolumn{6}{|l|}{ CSG } \\
\hline $\mathrm{IM}(\mathrm{n}=8)$ & $267.19 \pm 17.21$ & $101.83 \pm 5.53$ & $98.15 \pm 0.59$ & $13.73 \pm 0.44$ & $43.25 \pm 2.95$ \\
\hline $8 \mathrm{~W}(\mathrm{n}=9)$ & $488.89 \pm 28.03$ & $88.18 \pm 2.98$ & $96.54 \pm 1.61$ & $14.59 \pm 0.92$ & $43.38 \pm 3.78$ \\
\hline $12 \mathrm{~W}(\mathrm{n}=12)$ & $549.33 \pm 36.96$ & $87.01 \pm 4.06$ & $96.65 \pm 0.63$ & $15.00 \pm 0.55$ & $44.91 \pm 1.62$ \\
\hline \multicolumn{6}{|l|}{ SOG } \\
\hline $8 \mathrm{~W}(\mathrm{n}=11)$ & $541.63 \pm 29.67$ & $104.89 \pm 6.06$ & $97.92 \pm 0.69$ & $13.92 \pm 1.09$ & $41.70 \pm 3.29$ \\
\hline $12 \mathrm{~W}(\mathrm{n}=12)$ & $585.34 \pm 47.87$ & $100.98 \pm 7.25$ & $98.36 \pm 0.76$ & $13.62 \pm 1.42$ & $43.19 \pm 2.12$ \\
\hline
\end{tabular}

TABLE E2. Right ventricular hypertrophy index and general hemodynamics before and after shunt closure

\begin{tabular}{|c|c|c|c|c|}
\hline \multirow[b]{2}{*}{ Variable } & \multirow[b]{2}{*}{ RVHI ( $\%)$} & \multicolumn{3}{|c|}{ Hemodynamics (before/after shunt occlusion) } \\
\hline & & RVSP (mm Hg) & PASP (mm Hg) & mPAP (mm Hg) \\
\hline \multicolumn{5}{|l|}{ RPALG } \\
\hline $8 \mathrm{~W}(\mathrm{n}=8)$ & $32.83 \pm 4.79$ & $33.62 \pm 9.53$ & $31.77 \pm 8.19$ & $20.85 \pm 4.51$ \\
\hline $12 \mathrm{~W}(\mathrm{n}=8)$ & $35.39 \pm 6.04$ & $40.51 \pm 8.37$ & $38.55 \pm 5.76$ & $23.39 \pm 5.40$ \\
\hline \multicolumn{5}{|l|}{ LCSG } \\
\hline $8 \mathrm{~W}(\mathrm{n}=8)$ & $33.87 \pm 4.41$ & $34.15 \pm 8.34 / 28.46 \pm 5.38$ & $32.15 \pm 4.58 / 27.31 \pm 6.29$ & $22.46 \pm 4.38 / 19.50 \pm 3.29$ \\
\hline $12 \mathrm{~W}(\mathrm{n}=8)$ & $37.31 \pm 5.39$ & $39.69 \pm 5.94 / 31.81 \pm 7.14$ & $37.90 \pm 7.41 / 30.28 \pm 3.32$ & $27.13 \pm 6.67 / 21.38 \pm 4.57$ \\
\hline \multicolumn{5}{|l|}{ CSG } \\
\hline $\mathrm{IM}(\mathrm{n}=8)$ & $30.10 \pm 3.17$ & $45.72 \pm 6.02 / 37.85 \pm 7.05$ & $43.90 \pm 5.05 / 33.71 \pm 1.75$ & $25.92 \pm 1.43 / 22.62 \pm 2.12$ \\
\hline $8 \mathrm{~W}(\mathrm{n}=8)$ & $51.83 \pm 7.41$ & $61.62 \pm 11.33 / 52.30 \pm 11.21$ & $57.77 \pm 11.81 / 50.17 \pm 11.33$ & $35.03 \pm 7.78 / 32.65 \pm 6.59$ \\
\hline $12 \mathrm{~W}(\mathrm{n}=10)$ & $62.88 \pm 7.65$ & $67.51 \pm 11.20 / 63.76 \pm 8.53$ & $62.55 \pm 11.52 / 58.50 \pm 7.10$ & $36.84 \pm 4.81 / 34.31 \pm 2.95$ \\
\hline \multicolumn{5}{|l|}{ SOG } \\
\hline $8 \mathrm{~W}(\mathrm{n}=11)$ & $29.45 \pm 1.29$ & $27.62 \pm 3.24 / 29.07 \pm 2.36$ & $24.92 \pm 3.23 / 26.01 \pm 2.57$ & $18.09 \pm 1.43 / 19.21 \pm 1.74$ \\
\hline $12 \mathrm{~W}(\mathrm{n}=12)$ & $28.36 \pm 2.48$ & $28.31 \pm 1.73 / 27.91 \pm 3.17$ & $26.07 \pm 2.47 / 27.39 \pm 3.16$ & $17.75 \pm 1.59 / 18.53 \pm 2.12$ \\
\hline
\end{tabular}

